\title{
Evaluation of central auditory discrimination abilities in older adults
}

\section{Claudia Freigang ${ }^{1}$, Lucas Schmidt $^{2}$, Jan Wagner ${ }^{3}$, Rahel Eckardt $^{4}$, Elisabeth Steinhagen-Thiessen ${ }^{4}$, Arne Ernst $^{3}$ and Rudolf Rübsamen ${ }^{*}$ *}

\author{
1 Faculty of Bioscience, Pharmacy and Psychology, University of Leipzig, Leipzig, Germany \\ 2 Faculty of Mathematics and Natural Sciences I, Humboldt University Berlin, Berlin, Germany \\ ${ }^{3}$ Department Otolaryngology, Unfallkrankenhaus Berlin, Berlin, Germany \\ ${ }^{4}$ Forschungsgruppe Geriatrie am Evangelischen Geriatriezentrum Berlin, Charité - Universitätsmedizin Berlin, Berlin, Germany
}

Edited by:

Sige Zou, National Institute on Aging, USA

\section{Reviewed by:}

Josef Syka, Academy of Sciences of the Czech Republic, Czech Republic Daniel Fogerty, Indiana University, USA

*Correspondence:

Rudolf Rübsamen, Faculty of Bioscience, Pharmacy and Psychology, University of Leipzig, Talstrasse 33, 04103 Leipzig, Germany.

email: rueb@rz.uni-leipzig.de
The present study focuses on auditory discrimination abilities in older adults aged $65-89$ years. We applied the "Leipzig inventory for patient psychoacoustic" (LIPP), a psychoacoustic test battery specifically designed to identify deficits in central auditory processing. These tests quantify the just noticeable differences (JND) for the three basic acoustic parameters (i.e., frequency, intensity, and signal duration). Three different test modes [monaural, dichotic signal/noise (s/n) and interaural] were used, stimulus level was $35 \mathrm{~dB}$ sensation level. The tests are designed as three-alternative forced-choice procedure with a maximum-likelihood procedure estimating $p=0.5$ correct response value. These procedures have proven to be highly efficient and provide a reliable outcome. The measurements yielded significant age-dependent deteriorations in the ability to discriminate single acoustic features pointing to progressive impairments in central auditory processing. The degree of deterioration was correlated to the different acoustic features and to the test modes. Most prominent, interaural frequency and signal duration discrimination at low test frequencies was elevated which indicates a deterioration of time-and phase-dependent processing at brain stem and cortical levels. LIPP proves to be an effective tool to identify basic pathophysiological mechanisms and the source of a specific impairment in auditory processing of the elderly.

Keywords: age-related hearing loss, central auditory processing disorders, psychoacoustic test tool, temporal processing

\section{INTRODUCTION}

It is well documented that hearing sensitivity is decreased in older adults ( $\geq 60$ years of age; Brant and Fozard, 1990; Gates et al., 1990; Cruickshanks et al., 1998; Mazelová et al., 2003) a condition termed presbycusis (Schuknecht, 1955). Aging subjects often not only suffer from progressive deterioration of high frequency signal detection, but also from a gradual decline in speech perception, especially in noisy, and reverberant environments (CHABA, 1988; Dubno et al., 1997; Divenyi et al., 2005). According to the CHABA (1988) report, there are three hypotheses with regard to age-related deteriorations in speech perceptions: (i) the peripheral hypothesis assuming a cochlear dysfunction as the cause for impaired central processing (Schuknecht, 1955), (ii) the central auditory hypothesis stressing dysfunctional auditory brainstem and cortical processing, and (iii) the cognitive hypothesis assuming a decline in superordinate neuronal processing (i.e., attentional control and working memory; review: Humes, 1996; Pichora-Fuller, 1997). The central auditory hypothesis is supported by the fact that adult hearing impaired subjects (even when equipped with digital hearing aids) and older people with (age-matched) normal hearing often complain about verbal communication problems, i.e., they suffer from impairments in speech- and especially in speech in noise comprehension (Humes, 1996; Pichora-Fuller and Souza, 2003; Divenyi et al., 2005; Tremblay et al., 2007). This is clear evidence of pathophysiological changes in central auditory processing. Central auditory processing is dependent on temporal (time- and phase-dependent) and spatial integration of monaural and binaural sounds (e.g., Blauert, 1997). The discrimination of sounds deviating in signal duration, intensity, and frequency is indicative of the status of these integrative processes and hence can be used for the evaluation of the influence of age and/or hearing loss on central auditory processing. However, the impact of impaired cognitive attentional processes on central auditory processing mechanisms cannot be excluded in older adults (Craik and Salthouse, 2000), especially deficits in working memory and attentional top-down modulations (Gazzaley et al., 2005). Psychoacoustic discrimination tests aiming at signal difference limens of basic acoustic parameters are regularly used as a measure of central auditory processing abilities. To date, a number of studies quantified frequency, intensity, and duration discrimination in older adults which to different degrees covaried with hearing sensitivity (e.g., duration and frequency: Abel et al., 1990; frequency: König, 1957; Turner and Nelson, 1982; Freyman and Nelson, 1991; Buss et al., 2004; He et al., 2007; intensity and frequency: He et al., 1998).

Most discrimination studies compared the processing of two of the three basic acoustic parameters, e.g., frequency and intensity discrimination or frequency and duration discrimination. Furthermore, previous psychoacoustic studies were based on twoalternative forced-choice (2AFC) or the constant stimuli "yes/ no" paradigm, which both have high guessing rates as inherent 
properties. To collect a comprehensive set of data, we applied the "Leipzig inventory for patient psychoacoustic" (LIPP), a psychoacoustic test tool that comprises frequency, intensity, and duration discrimination tests based on a three-alternative forced-choice (3AFC) procedure and a maximum-likelihood paradigm. This approach has a number of advantages: (1) It is time-saving, since a relatively small number of trials is needed to estimate a respective threshold value (Leek, 2001). Test time is a critical factor in studying older adults, because subjects tend to have shorter attention spans (e.g., Salthouse, 1991). Psychoacoustic tests are typically time consuming, but with the adaptive maximumlikelihood procedure the number of trails needed to estimate a threshold is reduced. (2) The 3AFC paradigm facilitates a more precise measure of the subject's performance because the guess probability is reduced (He et al., 2007). (3) Since the tests do not make use of speech material and do not require verbal responses by the subjects, the results do not depend on the language competence of the subjects. LIPP has demonstrated to be a suitable test procedure and was already applied in healthy subjects aged 20-59 years (Bungert-Kahl et al., 2004), in patients with unilateral brain lesions (Biedermann et al., 2008), and in children with central auditory processing disorders and healthy children (Ludwig et al., 2009). Results from previous studies showed that with the help of LIPP a differentiation of declined processing at brainstem or diencephalic/telencephalic levels is possible. This illustrates that the application of LIPP generates data which can be directly compared across subjects with different native languages and different cognitive statuses and also across subjects of different age groups.

\section{MATERIALS AND METHODS SUBJECTS}

Subjects above the age of 65 years were recruited from the Seniorenuniversität (Seniors University), a facility of the Charité - Universitätsmedizin Berlin, at the Unfallkrankenhaus Berlin (UKB), and at a retirement home (Pflegeheim Hedwig, Berlin - Karlshorst) in Berlin. From 69 subjects tested, 59 were included in this study (65-89 years: 28 female and 31 male, mean $=73.3$ years, $\mathrm{SD}=6.72$ ). Ten subjects were excluded from the study due to incomplete data acquisition. All participants accomplished an anamnesis questionnaire about handedness, personal estimation of hearing ability, perception, and comprehension of speech, auditory localization, and the incidence of tinnitus as well as the mini-mental status examination (MMSE; Folstein et al., 1975). All subjects scored above 27 which served as evidence that they did not suffer from dementia. Additionally, we conducted an otoscopic examination, which showed no evidence of tympanic membrane abnormality. The data from the young age group 20-29 years was taken from the study by Bungert-Kahl et al. (2004). The young group was composed of 16 healthy normal hearing subjects ( 8 female and 8 male, mean $=23.8$ years, $\mathrm{SD}=3.5)$. Only old subjects were grouped into low or high performers based on their performance level (median split, Nagel et al., 2009). The experimental procedures conform to The Code of Ethics of the World Medical Association (Declaration of Helsinki) and were approved by the ethics review board of the University of Leipzig.

\section{STIMULI AND PARADIGM}

The psychoacoustic test procedures were based on the use of a psychoacoustic setup [RP2.1, Tucker-Davis-Technologies (TDT, System3)] controlled by custom-written MATLAB 6.3 scripts. Monaural and binaural stimuli were presented through headphones (Beyerdynamics, DT 770 Pro) at $35 \mathrm{~dB}$ SL (sensation level), i.e., at constant above-threshold levels. This level guaranteed a moderate loudness of the stimuli, but excluded any crosstalk from one headphone transducer to the contralateral ear, which could have obscured the results. Stimulus duration was $250 \mathrm{~ms}$ including $10 \mathrm{~ms}$ cosine-square ramps; interstimulus interval was $750 \mathrm{~ms}$. Time required for a complete data acquisition in a subject was about $2-3$ h.

\section{PURE-TONE AUDIOGRAM}

To quantify the subject's frequency-specific cochlear signal detection, the pure-tone hearing thresholds were obtained for each ear at $0.125 / 0.25 / 0.5 / 1.0 / 2.0 / 4.0$ and $8.0 \mathrm{kHz}$ as well as the detection thresholds for a Gaussian noise [0.1-20.0 kHz; yes/no (heard/ not heard) paradigm].

\section{EXPERIMENTAL PARADIGM}

Tests were designed to scrutinize the just noticeable differences (JNDs) for tone bursts differing in the basic acoustic features: frequency, intensity, or signal duration. Three different test modes were used: (1) monaural tests, i.e., monaural signal presentation at either ear, (2) dichotic signal/noise ( $\mathrm{s} / \mathrm{n}$ ) tests, i.e., dichotic presentation of $s / n$ pairs, and (3) interaural tests, i.e., binaural signal presentation with interaural signal differences (see Biedermann et al., 2008 for an evaluation of the percept related to the different stimulus modes).

The 3AFC method was used in all discrimination tests. Subjects were asked to differentiate between two reference signals and one deviant signal that differed in a single acoustic feature with the position of the deviant signal randomly altered within the stimulus triplet. Such tests are manageable even without the subjects being aware of the specific acoustic property that was varied during testing. The tests yielded a reliable outcome as long as the subjects were able to apply the concept of "same" and "different" to three successively presented acoustic signals and to indicate with some consistency, the one detected as different. Such a standardized test design was chosen to minimize the amount of instructions necessary to explain every single test to the subjects. Previous evaluations of the tests in patients from neurology disclosed the strength of this procedure (Biedermann et al., 2008), which enabled reliable assessment of auditory perception even in subjects that potentially have difficulties in comprehending test instructions (see also BungertKahl et al., 2004 for more details on the test procedures).

Psychometric functions were obtained by applying a maximumlikelihood method and estimation of a logistic function Eq. 1:

$p(t)=\frac{x_{4}+\left(x_{3}\right)}{\left(1+\mathrm{e}^{-\left(t-x_{1}\right) * x_{2}}\right)}$

where $p(t)$ is the probability of the correct response for a given $\Delta$ signal (i.e., $\Delta$ frequency, $\Delta$ intensity, $\Delta$ duration), $x_{1}$ is the range of values included in the estimation of the logistic function, $x_{2}$ 
describes the shape of the logistic function $\left(x_{2}=4 * \operatorname{slope}\left(x_{1}\right) / x_{3}\right)$, $x_{3}$ described the range between minimum $\Delta$ signal and maximum $\Delta$ signal (assumed as 1.0) of the function and $x_{4}$ the minimum $\Delta$ signal at one-third.

The psychometric function is described as a logistic function that is shaped by the maximum-likelihood procedure. The stimulus scale is important for applying the maximum-likelihood procedure to determine the parameters as well as the shape of the psychometric function. We were able to apply the stimulus scale from Bungert-Kahl et al. (2004) since test equipment and test procedure was the same (see Table 1 for details). The preliminary shape of the logistic function was calculated after eight trials with predefined initial step sizes. The final step size was applied and adaptively changed according to the individual performance after trial number eight. Final threshold and psychometric function were determined after the estimate asymptote at a respective threshold value (at about 22 trials). The 50\% threshold of the psychometric function was taken to compare values of the present study with the normative data of young subjects collected by Bungert-Kahl et al. (2004).

There are a number of features which make these tests specifically suitable for the evaluation of central auditory processing capacity: In all test modes the subjects have to compare an internalized percept associated with three acoustic events after the whole of the stimulus triplet has been presented: Frequency. The deviant signal differed in its frequency, which was always higher than the standard frequency. Intensity. The intensity of the deviant signal was always higher than the standard intensity. Duration. The deviant signal was shorter than the standard signal.

\section{TEST MODES}

(1) In the monaural tests the percept conveys deviant information about a single acoustic feature, frequency, intensity, or signal duration. The discrimination limens (DL) for the respective features indicate the capacity of central auditory processing (which self-evidently is also limited by the constraints of middle ear signal transmission and cochlear signal transduction). The monaural test mode depends on central auditory processing and the cognitive capacity to create an internal representation of the three signals and to decide which signal differed in the respective parameter. Figure $\mathbf{1 A}$ depicts the characteristics of the signal triplet differing in the acoustic feature signal duration. In this example, the signal triplet is presented at the right ear. Deviant signals were also altered in signal frequency or signal intensity.

(2) The dichotic s/n tests were - with regard to the test systematic - an extension of the monaural tests, as here the signal presentation to one ear was paired with bandpass noise bursts $(0.1-20 \mathrm{kHz} ; 250 \mathrm{~ms})$ presented to the other ear (Figure 1B).
The noise is intended to interfere with processing of the test signals, causing central auditory masking (Zwislocki, 1972; Mills et al., 1996). Same as for monaural tests, the performance in the dichotic tests also strongly depend on central auditory processing. Biedermann et al. (2008) used these tests and examined central auditory processing in patients with unilateral lesions. Patients with unilateral lesions were able to perform dichotic $s / n$ tests when the signal triplet was presented ipsilateral to the lesion. With the signal triplet presented contralateral to the lesion, however, performance was significantly impaired. These findings suggest that dichotic $s / n$ tests aim at cortical processing stages. Figure 2 shows an example for duration discrimination; alternatively, deviant signals were also altered in signal frequency or signal intensity.

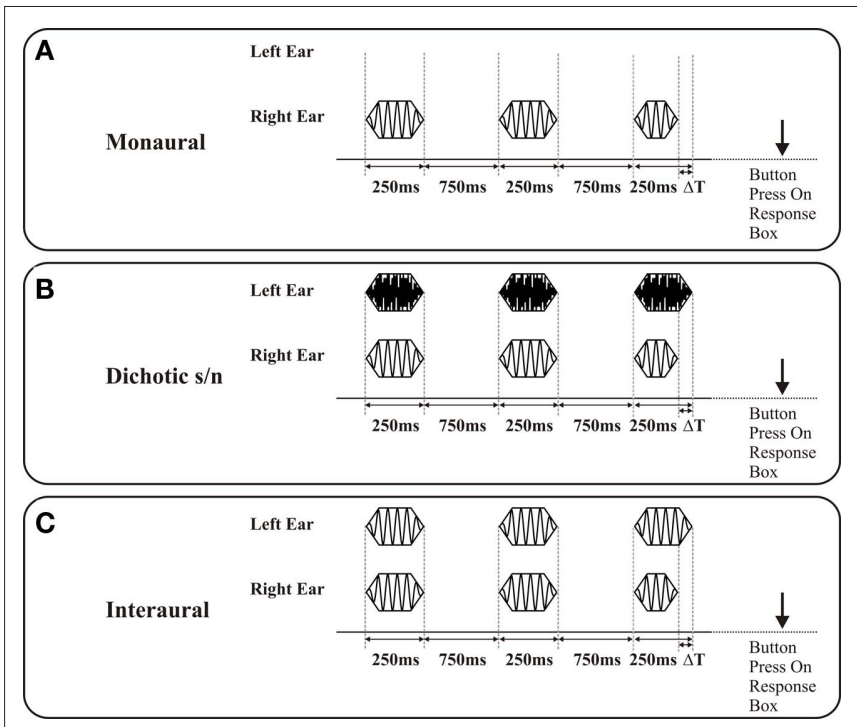

FIGURE 1 | Depiction of signal presentation on discrimination of differences in signal duration (signal duration $250 \mathrm{~ms}$; interstimulus interval $750 \mathrm{~ms}$ ): The duration of the deviant is shorter than the reference signals and systematically prolonged $(\Delta T)$ starting with an initial value of $\Delta 120 \mathrm{~ms}$, the position of the deviant in the triplet is randomized in successive presentations. The subject is asked to indicate the position of the deviant signal in the stimulus triplet by pressing a button on a response box. (A) Monaural presentation: Signals are presented either to the right or the left ear; the graph shows a signal triplet presented to the right ear. (B) Dichotic signal/noise presentation: Signal triplets are presented either to the right or the left ear (in the present example to the right ear). A triplet of noise bursts are concurrently presented to the respective other ear (in the present example to the left ear, noise burst duration $250 \mathrm{~ms}$ ). (C) Interaural presentation: For the reference condition, the signals presented to both ears were identical in frequency. The deviant signal on the right ear coincides with a standard signal on the left ear. In the case of duration discrimination a movement of the sound from the intracranial on-center position to the standard signal is perceived.

Table 1 | Test parameter overview.

\begin{tabular}{lllllll}
\hline Discrimination test & Test variable & Standard frequency $(\mathbf{k H z})$ & Initial value & Mode of change & Initial step size & Final step size \\
\hline Frequency & $\Delta \mathrm{F}(\mathrm{Hz})$ & $0.5 / 1.0 / 2.0$ & $1000 \mathrm{~Hz}$ & Multiplicative & 2.0 & 1.1 \\
Intensity & $\Delta \mathrm{l}(\mathrm{dB})$ & $0.5 / 1.0 / 2.0$ & $20 \mathrm{~dB}$ & Additive & $5 \mathrm{~dB}$ & $2 \mathrm{~dB}$ \\
Duration & $\Delta \mathrm{T}(\mathrm{ms})$ & $0.5 / 1.0 / 2.0$ & $120 \mathrm{~ms}$ & Additive & $10 \mathrm{~ms}$
\end{tabular}




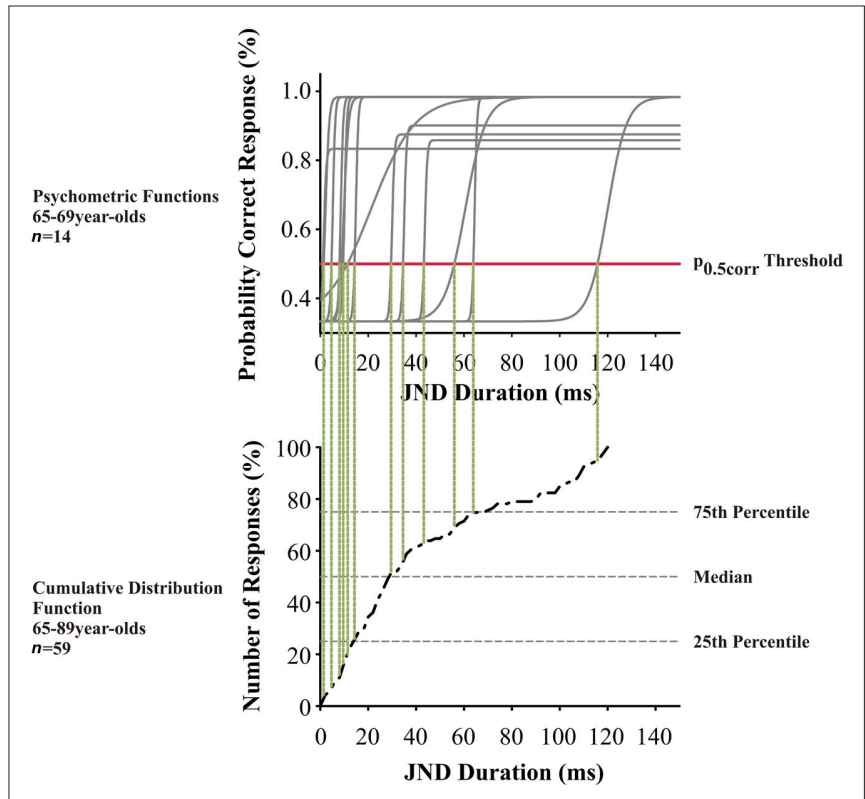

FIGURE 2 | Top panel: Psychometric functions from the left ear of 65- to 69-year-old subjects $(n=14)$ for interaural duration discrimination at $500 \mathrm{~Hz}$. Each gray solid line indicates the psychometric function of one subject; the red horizontal line indicates the level of the $p_{0.5 c o r r}$ values. Lower panel: Cumulative distribution for interaural duration discrimination at $500 \mathrm{~Hz}$ in 65- to 89-year-old subjects. The $p_{0.500 r}$ thresholds from left and right ears from all subjects ( $n=59)$ were included; the respective values of the above are indicated by vertical dotted lines.

(3) In all interaural tests, the presentation of identical signals to the two ears constituted the reference condition (diotic stimulation). For acquisition of interaural frequency difference limens the test variable was $\Delta$ frequency at the two ears (both starting at cosine), for interaural intensity differences the test variable was $\Delta$ intensity, and for interaural duration difference $\Delta$ signal duration. In case of $\Delta$ duration the signals at the right and the left ear started at the same time, but the deviant signal was shorter (see Figure 1C). Common to all these interaural mismatches is the induction of sound percept that appear intracranially lateralized on an interaural axis as compared to on-center percept connected to the reference stimuli. Lateralization appears static for $\Delta$ intensity and "moving" for $\Delta$ frequency and $\Delta$ signal duration, i.e., the signal moves from the on-center position toward the longer signal and swaps around the on-center position at different frequencies, respectively. Interestingly, the naive subject is not able to identify which acoustic feature is varied in any of the interaural tests (see Biedermann et al., 2008 for a more detailed evaluation of the percept related to the different stimulus modes).

\section{STATISTICAL ANALYSES}

The threshold at the $p=0.5$ correct response value $\left(p_{0.5 \text { corr }}\right)$ from each psychometric function was estimated for ear and subject (Figure 2). Values from both ears were pooled (since tests did not show differences between the two ears) and included into the calculation of the respective cumulative distribution function, where each data point represents the $p_{0.5 c o r r}$ threshold of one ear. We applied a repeated measurements analysis of variance (ANOVA) with the factors test mode (three levels: monaural, dichotic $s / \mathrm{n}$, and interaural), frequency (three levels: $0.5 ; 1.0$; and $2.0 \mathrm{kHz}$ ) and age, including a Greenhouse-Geisser correction. After that, a two-tailed Student's $t$-test was used to compare test modes at defined frequencies. Correlation analysis between hearing threshold and threshold values were conducted with the Pearson product-moment correlation coefficient $r$ (Pearson's $r$ ).

\section{RESULTS}

\section{SUBJECTS}

From the urban population of Berlin (Germany) 69 subjects were tested, and data from 59 subjects ( 30 males and 29 females) were included in this study. The otoscopic examination showed no evidence of tympanic membrane abnormality. Ten subjects were excluded from the study, because they were not able to complete a minimal required number of subtests during the scheduled test time.

\section{PURE-TONE AUDIOGRAM}

The data show a clear deterioration of hearing sensitivity with increasing age $\left(F_{6,70}=211.992, p=0.000\right)$ and dependencies on test frequency $\left(F_{6,70}=103.340, p=0.000\right.$; Figure 3A). When referenced against the hearing thresholds of the 20- to 29-year-old subjects, the graded frequency-specific elevation in threshold values was $10-35 \mathrm{~dB}$ between 0.125 and $2.0 \mathrm{kHz}$ and $40-55 \mathrm{~dB}$ between 4.0 and $8.0 \mathrm{kHz}$ (Figure 3B). This indicates the typical pattern of presbycusis, with hearing thresholds being more increased at high than at low frequencies. Some of the subjects aged 65-89 years were not able to detect an $8.0-\mathrm{kHz}$-tone burst at a maximum intensity of $101 \mathrm{~dB}$ SPL. The data also show an apparent increase in intersubject variability with increasing age as seen in the interquartile distances of threshold values most prominent at high frequencies.

\section{DISCRIMINATION TASKS}

The acquired discrimination thresholds for the 65- to 89-year-old subjects were screened for ear and gender effects. Since no significant or systematic differences between left and right ear or male and female subjects were found, the data were pooled for further analysis over both ears and sexes for the young adults and old adults, respectively.

\section{INTENSITY DISCRIMINATION}

The intensity discrimination in both age groups showed characteristic differences between the three test modes $\left(F_{4,70}=30.820\right.$, $p=0.000$ ), but only minor changes for the different test frequencies within the respective presentation modes (Figure 4). As a general trend (also apparent in the other discrimination tests described below) it can be stated that the threshold values in the elderly were significantly higher than in young adults $\left(F_{4,70}=15.450, p=0.000\right)$. Still, it is conspicuous that the 25th percentiles in the different age groups differed only slightly $(0.3-2.2 \mathrm{~dB})$, which indicates that the best performance in the elderly is in the same range as the respective performance in young adults. However, in all tests the 75 th percentile in the elderly was two to three times $(4-5 \mathrm{~dB})$ 

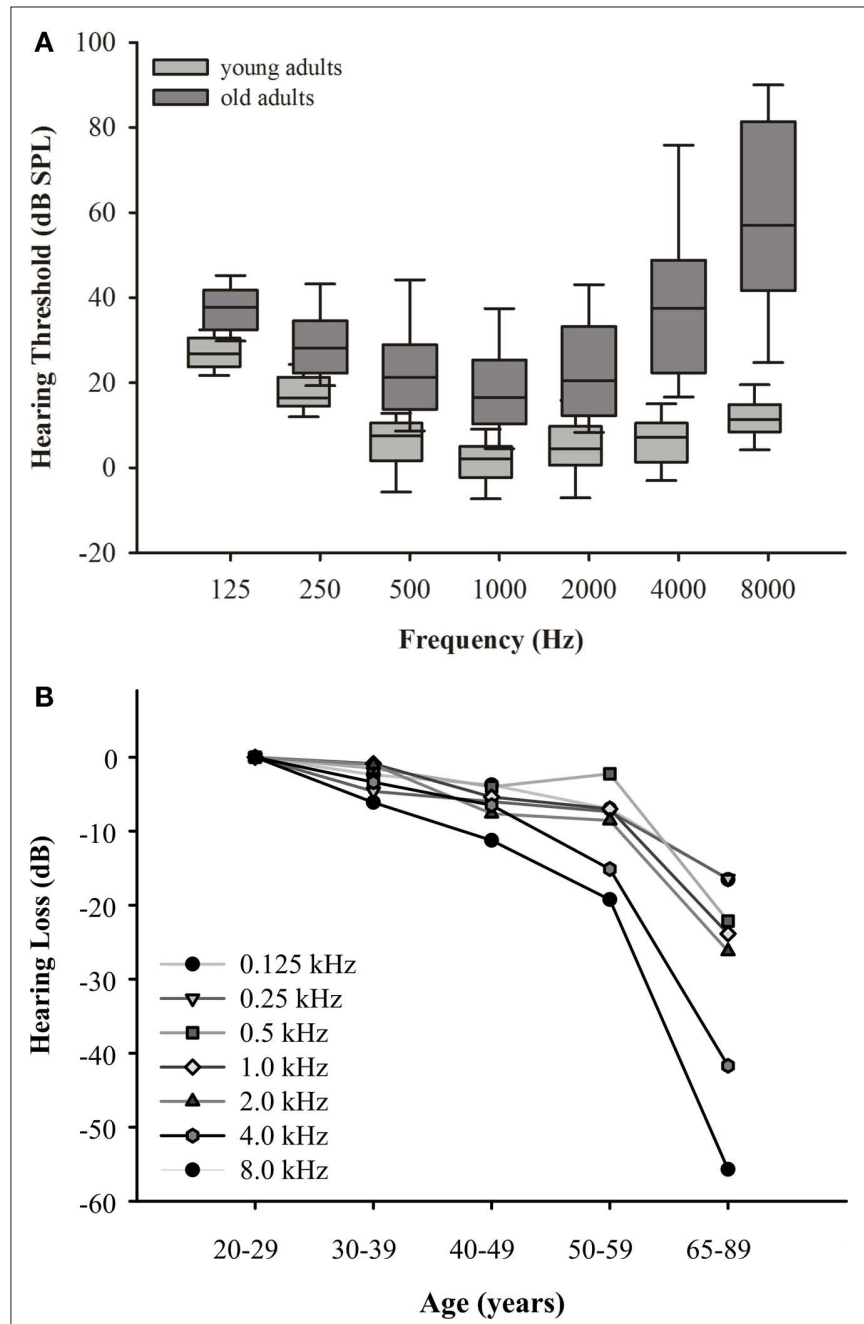

FIGURE 3 | (A) Hearing thresholds for the age groups 20-29 $(n=16)$ and 65-89 $(n=59)$ years. Box plots show results at different test frequencies with medians, 25th/75th quartiles, and 95th/5th percentiles; different gray scales indicate data from the respective age groups. Data of 20- to 29-year-old subjects are from Bungert-Kahl et al. (2004). Note the elevation of threshold values and (most prominent at high frequencies) the increase in interindividual variability with age. (B) Mean hearing loss across age groups 20-29, 30-39, 40-49, 50-59 (data from Bungert-Kahl et al., 2004), and 65-89 years (present study). Hearing loss was mostly present at frequencies above $2.0 \mathrm{kHz}$.

higher than that of the young adults. This results in a much wider distribution of the interquartile range in the elderly which covers two to three times the range of the young adults. Consequently, the elderly also showed elevated median values. The ranges between the median values and the respective 25 th and 75 th percentiles were quite similar which indicates that the distributions were not biased to one side. While the different test modes yielded statistically different threshold values in young adults, with the interaural tests showing the highest values, in old adults there were no such differences. The correlation of hearing thresholds and threshold values revealed a significant dependency at 1.0 (Pearson's $r=0.183$, $p=0.03$ ) and $2.0 \mathrm{kHz}$ (Pearson's $r=0.246, p=0.011$ ) of monaural intensity discrimination.

\section{FREQUENCY DISCRIMINATION}

Frequency discrimination in the older adults was significantly different from young adults $\left(F_{4,70}=31.373, p=0.000\right.$; Figure 5). It has to be considered in these tests is that across the different age groups the performance additionally depended on test frequency $\left(F_{4,70}=6.461\right.$, $p=0.006)$. The ANOVA also revealed significant interaction of frequency and age $\left(F_{4,70}=8.010, p=0.001\right)$. Similar to intensity discrimination, the 25 th percentile of the young and elderly were in a close range $(0.2-2 \%)$. In contrast, the 75 th percentile of the $65-$ to 89 -year-old subjects were about two to seven times larger than those of the 20- to 29-year-old subjects. Consistently, the median values were likewise elevated, but stayed more close to the 25 th percentile. Thus the high performers were less variable in their performance than the low performers. Consequently, about $60 \%$ of the subjects showed frequency discrimination in a narrow range of 3-4\% $(1-60 \mathrm{~Hz})$, while about $40 \%$ had thresholds spanning a wide frequency range from 5 to $50 \%(16-1860 \mathrm{~Hz})$. Monaural frequency discrimination thresholds were significantly dependent on hearing thresholds at 0.5 (Pearson's $r=0.217, p=0.018$ ) and $1.0 \mathrm{kHz}$ (Pearson's $r=0.223, p=0.017$ ). Dichotic $s / n$ frequency discrimination thresholds were only dependent on hearing threshold at $1.0 \mathrm{kHz}$ (Pearson's $r=0.224, p=0.008$ ).

Differences between the age groups 20-29 and 65-89 years were smallest in interaural tests. Here, high performers yielded thresholds equivalent to the young at $0.5 \mathrm{kHz}$. The results from the interaural test at $0.5 \mathrm{kHz}$ were significantly different from monaural $\left(t_{58}=5.114, p=0.000\right)$ and dichotic frequency discrimination $\left(t_{58}=4.225, p=0.000\right)$. At 1.0 and $2.0 \mathrm{kHz}$, threshold values were close to those in the monaural tests although they showed a reduced overall variance (Figure 5). The largest variability was found in the dichotic s/n tests, but the median values were not significantly different from those of the respective monaural tests.

\section{DURATION DISCRIMINATION}

As described for the tests above, thresholds for signal duration discrimination were strongly age-dependent $\left(F_{4,70}=25.078\right.$, $p=0.000$; Figure 6). Furthermore, for these tests performances of subjects aged 65-89 years showed particularly high variability as well as a strong deviation from the results obtained in the younger adults. Unlike in the previous tests, and specifically for the monaural and for the dichotic s/n stimulation, threshold values in the older age group showed lesser skewed distributions, i.e., $25 \%$ of the threshold values below and $25 \%$ above the median cover mostly equivalent difference ranges. The 25 th percentiles covered the range of 20-50 ms and the respective 75th percentiles $35-90 \mathrm{~ms}$. Additionally, monaural threshold values at $0.5 ; 1.0$; and $2.0 \mathrm{kHz}$ were highly dependent on hearing thresholds; the same holds for dichotic $\mathrm{s} / \mathrm{n}$ discrimination thresholds were 0.5 and $1.0 \mathrm{kHz}$ (see Table 2). Interaural duration discrimination thresholds did not covary with the hearing thresholds.

The threshold values in the interaural tests yielded a more uneven distribution: The high performers had thresholds which were close to those of the 20- to 29-year-old subjects, while the thresholds of the low performers were skewed to high values mostly covering the range of values measured in the monaural and dichotic $s / n$ tests. The low performers also reported problems in perceiving the stimuli as being lateralized. 

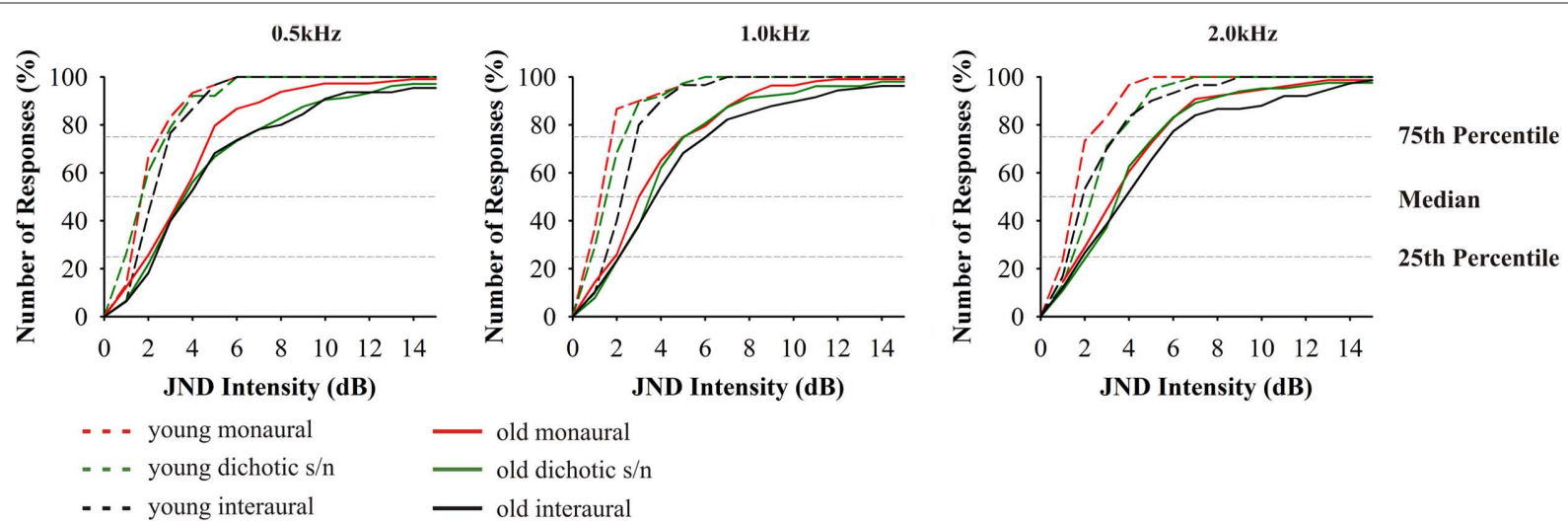

$$
\begin{array}{ll}
\text { - - - young monaural } & - \text { old monaural } \\
\text { - - - young dichotic } \mathrm{s} / \mathrm{n} & \quad \text { old dichotic } \mathrm{s} / \mathrm{n} \\
\text { - - - young interaural } & \quad \text { old interaural }
\end{array}
$$

FIGURE 4 | Cumulative distributions of JND of intensity discrimination. From left to right results for the test frequencies $0.5,1.0$, and $2.0 \mathrm{kHz}$. Red indicates: monaural stimulation of either ear; green: dichotic signal/noise stimulation; black: stimulation with interaural signal differences. The different age groups are indicated by the different line types: 20-29 $(n=16)$ long dashed lines,
65-89 ( $n=59$ ) solid. Data from 20- to 29-year-old subjects taken from Bungert-Kahl et al. (2004). Older subjects mostly performed poorer and showed a wider variation of threshold values than younger subjects. The best performances at the 25th percentiles were in a narrow range with the young, while the 75th percentiles show values elevated by $3-4 \mathrm{~dB}$.

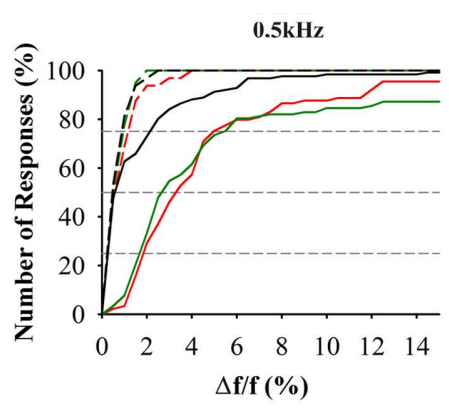

- - - young monaural

- - - young dichotic $\mathrm{s} / \mathrm{n}$

- - - young interaural
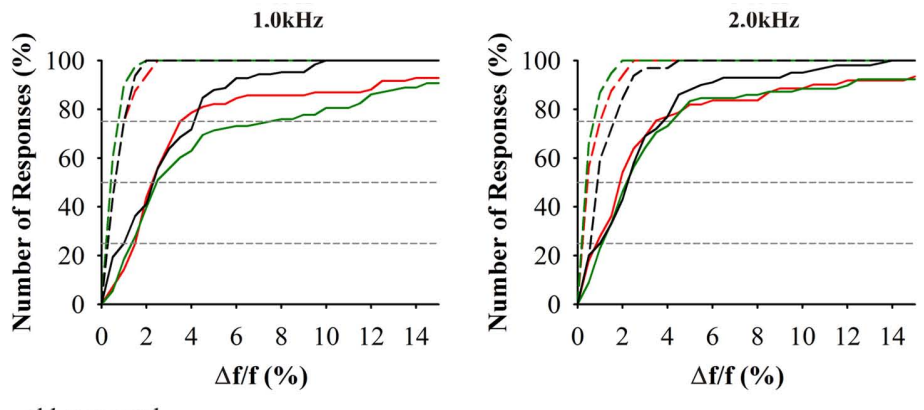

75th Percentile

Median

25th Percentile
FIGURE 5 | Cumulative distributions of JND of frequency discrimination; from left to right results for the test frequencies $\mathbf{0 . 5}, \mathbf{1 . 0}$, and $\mathbf{2 . 0} \mathbf{k H z}$. Since absolute values depend on the test frequency, JND are indicated as difference in percent from the respective test frequency. Red indicates: monaural stimulation of either ear; green: dichotic signal/noise stimulation; black: stimulation with interaural signal differences. The different age groups are indicated by the different line types: 20-29 ( $n=16)$ long dashed lines, $65-89(n=59)$ solid. Most prominently, performances depended on test alternative, frequency, and age. Variance increased in the elderly.

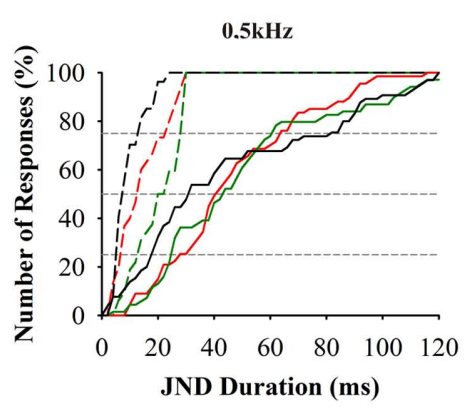

JND Duration (ms)
$1.0 \mathrm{kHz}$

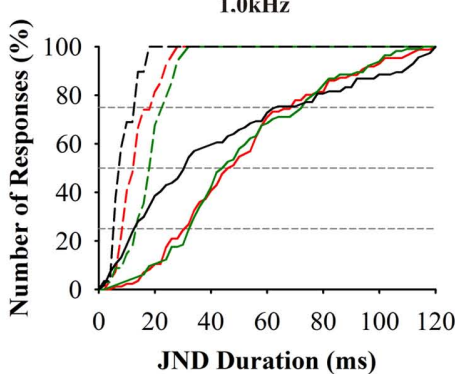

JND Duration (ms)

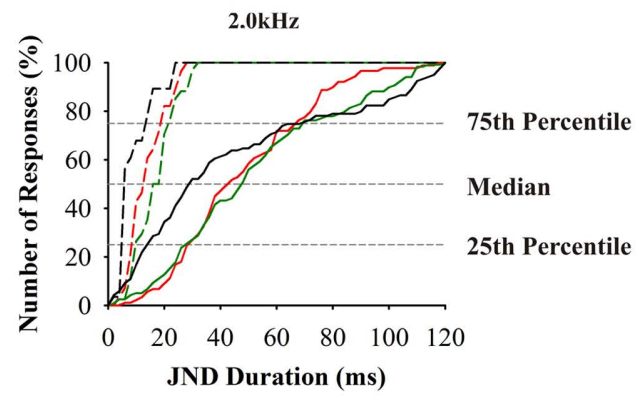

$\begin{array}{ll}\text { - - - young monaural } & - \text { old monaural } \\ \text { - - - young dichotic } \mathrm{s} / \mathrm{n} & - \text { old dichotic } \mathrm{s} / \mathrm{n} \\ \text { - - - young interaural } & \quad \text { old interaural }\end{array}$

FIGURE 6 | Cumulative distributions of JND of duration discrimination. From left to right results for the test frequencies $0.5,1.0$, and $2.0 \mathrm{kHz}$. Design of the graph like in Figure 4. The different age groups are indicated by the different line types: $20-29(n=16)$ dashed lines, 65-89 ( $n=59)$ solid. Data from 20- to 29-year-old subjects taken from Bungert-Kahl et al. (2004). Poorest performance was achieved in interaural tasks which showed the highest intersubject variability. 
Table 2 | Pearson's correlation coefficient Pearson's r of test mode (monaural and dichotic s/n) and hearing threshold $(0.5 ; 1.0$; and 2.0 kHz) of duration discrimination.

\begin{tabular}{|c|c|c|c|c|c|c|}
\hline Hearing threshold at: & & Monaural 0.5 kHz & Monaural 1.0 kHz & Monaural 2.0 kHz & Dichotic s/n 0.5 kHz & Dichotic s/n $1.0 \mathrm{kHz}$ \\
\hline \multirow[t]{2}{*}{$0.5 \mathrm{kHz}$} & Pearson's $r$ & 0.308 & l & l & 0.207 & I \\
\hline & $p$-value & 0.001 & & & 0.013 & \\
\hline $1.0 \mathrm{kHz}$ & $p$-value & & 0.001 & & & 0.000 \\
\hline $2.0 \mathrm{kHz}$ & Pearson's $r$ & l & / & 0.256 & l & / \\
\hline
\end{tabular}

Correlations lacking significance were marked with a slash and excluded from the table for clarity.

\section{HIGH AND LOW PERFORMERS ACROSS TEST MODES}

An overview of the data of all subjects shows that some performed low $(n=10)$ and others high $(n=13)$ across all test modes (see Materials and Methods for the respective distinction). Respective tests yielded no correlation of these results with the individual hearing threshold pointing to the fact that the performance of high and low performers did not reflect the status of inner ear signal transduction, but indeed the individual central auditory processing abilities.

\section{DISCUSSION}

The present study aims at a comprehensive data acquisition of auditory discrimination abilities in the elderly aged between 65 and 89 years. By applying a rich repertoire of tests, which helps to differentiate between the contribution of the auditory brainstem and respective diencephalic/forebrain levels to central auditory processing, this study conveys a broad data set on age-dependent changes of JNDs in the basic acoustic features, frequency, intensity, and signal duration. For this, we used the LIPP which originally was designed to identify deficits in central auditory processing in patients from neurology, who suffered from acquired brain lesions (Bungert-Kahl et al., 2004; Biedermann et al., 2008). The tests make (i) low demands on the cognitive state and attention span of the subjects, (ii) do not use speech material nor require verbal responses, and (iii) do not depend on the understanding of complicated test instructions. Additionally, (iv) these tests are independent of the patients' speech competence and of their cultural and language background. This makes it easier to compare the respective results across different countries and might help in the development of common therapeutic strategies.

\section{CONSIDERATION OF AUDITORY THRESHOLD}

For comparability of the results, all tests had to be performed at the same above-threshold values. Thus, the subjects' age-related gradual increase in hearing thresholds - specifically at frequencies $4.0 \mathrm{kHz}$ and above - were taken into account. Deterioration of high frequency sensitivity was already observed in the fifth decade of life (Bungert-Kahl et al., 2004; Johnson et al., 2005) and implacably worsens with age, so that the use of 4.0 and $8.0 \mathrm{kHz}$ test frequencies had to be excluded in subjects $\geq 70$ years of age. The frequency range employed in the present tests was restricted to $0.25-2.0 \mathrm{kHz}$, a frequency range which, in subjects up to the age of 89 years allowed stimulus presentation at $35 \mathrm{~dB}$ sensation level.

Across all age groups, the JNDs in the interaural tests showed no correlation with the subject's threshold. This suggests that the respective performance did not depend on the status of the peripheral auditory system, but clearly reflected central auditory processing abilities. In the monaural tests, however, the JNDs showed a weak correlation with hearing thresholds pointing to an influence of hearing threshold on the performance.

While researchers widely agree on the fact that elevation of hearing threshold is due to a decrease of the number of inner and outer hair cells (IHC, OHC; Soucek et al., 1986) and of auditory nerve fibers (Felder and Schrott-Fischer, 1995), the causes for the respective losses are still a matter of debate. What might play an important part is (i) a genetic predisposition and/or (ii) long-lasting hazardous noise exposure (e.g., loud working environment; review: Jennings and Jones, 2001), (iii) the use of ototoxic drugs, or (iv) metabolic diseases affecting capillary blood flow (e.g., diabetes mellitus; Celik et al., 1996). The multifactorial causes of high frequency hearing loss are reflected by the successive increase of the interquartile ranges of frequencyspecific threshold values with age. This highlights the importance of considering the individual hearing sensitivity when quantifying age-dependent JNDs of discrimination of acoustic features.

\section{DISCRIMINATION TASKS}

Across the different test modes with monaural, dichotic s/n, and interaural signal presentations, the JND for frequency, intensity, and signal duration increased with age, though there were prominent differences in discrimination thresholds related to the three acoustic features and to the test modes applied. Notably, the dispersion of the interquartile ranges increased with age. In some of the tests the distributions of the threshold values around the respective medians remained symmetrical, while in others they were skewed toward larger JNDs. Hearing threshold was not the overall predictive factor that contributed to the difference in performance between high and low performers (see Materials and Methods for the respective distinction). Hence, the functionality of individual central auditory processing abilities must be affected by age to some extent. Below, the respective aspects will be discussed in detail, but it should be mentioned that some influence of declined cognitive attentional processes cannot be excluded for data based on psychoacoustic tests. An earlier study reported that highperforming older adults benefit from activating compensatory cognitive networks to cope with demanding tasks, whereas low-performing older adults do not (Cabeza et al., 2002).

\section{Intensity}

Intensity discrimination thresholds in old adults were increased compared to young adults. The fact that JNDs only covaried in monaural intensity discrimination tasks with hearing thresholds, 
suggests that the central neural mechanisms underlying coding of intensity of interaural and dichotic s/n discrimination are affected by age-related changes. Monaural intensity discrimination is determined by the spike rate coding in the auditory nerve. A typical characteristic of presbycusis is the loss of the dynamic range in afferent auditory nerve firing progressing from high to low frequencies, referred to as "loudness recruitment" (Fowler, 1936; Dix et al., 1948; Marozeau and Florentine, 2007). Thus, it is not advisable to use frequencies $4.0 \mathrm{kHz}$ and above for the intensity discrimination tasks, since the results would not allow clear differentiation between peripheral and central causes of impaired intensity discrimination. When using frequencies $0.5-2.0 \mathrm{kHz}$, still the age-dependent threshold elevations have to be taken into account to perform different tests at the same sensation levels (Bungert-Kahl et al., 2004). There is a different aspect of the tests for intensity discrimination that renders them important for an overall evaluation of the subjects' central auditory processing: These tests are particularly easy to grasp and thus have proven to be good indicators for the subjects' capability to master the test procedure.

\section{Frequency}

The data on frequency discrimination are conspicuous in two ways: A very prominent increase in variability of JNDs in older subjects and a strikingly skewed distribution of JNDs toward large frequency differences. The present investigation same as previous studies have shown that a loss of hearing sensitivity is accompanied by a deterioration in monaural frequency discrimination (Turner and Nelson, 1982; He et al., 1998, 2007). Thus, the reduction in frequency selectivity does in part reflect the peripheral hearing loss. Also in the present data, monaural frequency discrimination thresholds were elevated in older adults. But beyond that, in the same subjects the JNDs for dichotic frequency discrimination tests showed an even greater elevation which might be explained by central masking (Zwislocki, 1972; Mills et al., 1996) and relate to the fact that older adults have major problems in detecting a sound in noise and to understand speech in noisy backgrounds (CHABA, 1988). Our current results suggest that frequency discrimination is based on different central auditory processing mechanisms across the different test modes with slightly different impacts of hearing loss on frequency discrimination performance.

Earlier studies on monaural frequency discrimination in older adults made use of different experimental procedures. He et al. (1998) estimated the psychometric function based on maximumlikelihood procedure and applied the 2AFC paradigm, while König (1957) used the constant stimuli approach. Our measurements yielded comparable results and also showed that $\Delta \mathrm{f} / \mathrm{f}$ is large at $500 \mathrm{~Hz}$ and decreases with increasing test frequency. Still, the respective absolute threshold values obtained here were significantly higher for both young and old adults which can be explained by the smaller guess probability (one-third) when using the 3AFC procedure. Notably, the relation between the threshold values in young adults in the study of He et al. (1998) and the present study is significantly smaller than the respective difference between the old adults. Thus, the present data is more indicative of actual changes in discrimination abilities, a fact which is important since intersubject variability increases strongly with age (Schneider et al., 1994).
The fact that $\Delta \mathrm{f} / \mathrm{f}$ values between young and old adults show a comparable relation across the different test frequencies suggests that the neuronal mechanisms of frequency discrimination are still preserved with the elevated thresholds being explainable by both, increased hearing thresholds and age-related changes in central auditory processing. With respect to the latter, two distinct processing mechanisms have to be differentiated: (i) the cochlear tonotopy indicative of spectral analysis and (ii) phase locking of discharges of auditory nerve fibers at frequencies $<2 \mathrm{kHz}$. Unless a subject is able of absolute pitch perception, monaural frequency discrimination can only be mastered by spectral analysis. In this respect, the present data do not allow to distinguish between peripheral and central contributions to frequency discrimination. But interaural frequency discrimination at low frequencies (i.e., $0.5 \mathrm{kHz}$ ) allows to evaluate how temporal processing contributes to frequency discrimination. Here, the underlying mechanism is the processing of converging afferent activities from both ears at brainstem levels (Blauert, 1997). This integration is highly dependent on precise temporal processing and in the respective frequency range auditory nerve fiber discharges are phase-locked to the period of the acoustic signals, and the respective activity converges onto nuclei of the superior olivary complex of the brainstem (Grothe et al., 2010). So, bilateral integrative frequency information is realized in form of "temporal processing." This is why the JNDs for interaural frequency discrimination are about one order of magnitude below the respective monaural values (Bungert-Kahl et al., 2004). In the present study, about $50 \%$ of the subjects (high performers) were able to reach thresholds similar to those in the young adults. In the same subjects (and also in the low performers) results from monaural and dichotic frequency discrimination were well above the thresholds of interaural tests. Hence, also in old subjects temporal processing contributes to frequency discrimination, at least at $0.5 \mathrm{kHz}$. The fact that at $1.0 \mathrm{kHz}$ the performance in the interaural tests is significantly worse, i.e., in the same range as performance in the monaural and dichotic tests, indicates a low-frequency shift of the cutoff of temporal resolution (auditory nerve fiber phase locking in low performers) contributing to frequency discrimination.

\section{Duration}

For all modes of duration discrimination and at all test frequencies, the respective thresholds in the old subjects were found to be elevated compared to the young adults. It was conspicuous that all duration discrimination tasks revealed the highest intersubject variability. Such high variability was also found in a variety of other studies investigating temporal signal processing (Durlach et al., 1981; Cranford et al., 1993; Schneider et al., 1994; Koehnke et al., 1995; Snell and Frisina, 2000). In the monaural and dichotic $\mathrm{s} / \mathrm{n}$ tests the JNDs of the low and high performers ranged between 60-120 and 5-30 ms, respectively. In the high performers, the thresholds in the interaural tests were below those of the monaural and dichotic $\mathrm{s} / \mathrm{n}$ tests. But, unlike for interaural frequency discrimination at $0.5 \mathrm{kHz}$, threshold values did not overlap with the range of young adults, i.e., the threshold values of the entire sample was evenly distributed across the stimulus scale. Additional tests revealed that the respective performance did not covary with the hearing threshold values of the subjects which is in agreement with 
results of earlier studies (e.g., Schneider et al., 1994; Fitzgibbons and Gordon-Salant, 1995, 1998; Gordon-Salant, 2005; Gordon-Salant et al., 2006; Heinrich and Schneider, 2006; Humes et al., 2010).

Duration discrimination relies on central auditory networks which comprise brainstem nuclei and subcortical and cortical networks (Kaukoranta et al., 1989; Belin et al., 2002). The hypotheses about the peculiarities of signal duration processing are based on electrophysiological studies, suggesting two different mechanisms: (i) The temporal response pattern of auditory nerve fibers and of neurons in central auditory nuclei encode by their activity period for the duration of the signal. (ii) Populations of neurons, encoding the onset and the offset of acoustic signals, establish the input to duration tuned neurons at the level of IC or auditory thalamus (Casseday et al., 2002). Possibly, both mechanisms jointly contribute and they might be backed up by (iii) the processing of interaural time differences (ITDs). When the focus is on cortical processing, duration discrimination depends on two different networks, the "attentional" (Mesulam, 1981, 1998; Belin et al., 2002) and "temporal network" (Ivry, 1996; Gibbon et al., 1997; Belin et al., 2002). The attentional network comprises activation of frontoparietal areas (BA 44, 45, 47, 6, and 40), whereas the temporal network relies on the interaction of the basal ganglia, thalamus, cerebellar hemispheres, and the right prefrontal cortex (Belin et al., 2002). Attentional processes depend on cognitive control which seems to be affected negatively by age, i.e., older adults fail to filter or inhibit irrelevant and extraneous information (Hasher and May, 1999). Consequently, even in easy tasks additional memory resources might be activated to recall previous sensory representations (Eckert et al., 2008). These networks might play the major role in monaural and dichotic s/n duration discrimination, since representations of three successive stimuli have to be compared post hoc for discrimination and the elevated threshold values in monaural and dichotic $\mathrm{s} / \mathrm{n}$ duration discrimination might be due to a reduced functionality of temporal and attentional networks. Ross et al. (2010) investigated auditory steady-state responses to sounds interrupted by gaps. High gamma and long-latency low-frequency activity should reflect object representation related processes and memory update, respectively. Old adults showed reduced phase locking and amplitude of high gamma activity as well as a longlatency low-frequency activity in older adults. These results point to a contribution of cortical networks to duration discrimination and reflect the impaired performances in older adults. The decrease in gamma-band activity might be caused by reduced glutamatergic neurotransmission, especially in parvalbumin neurons (Woo et al., 2010).

The conditions for interaural duration discrimination seem to be different. This is already reflected by the fact that any type of interaural temporal disparity creates percepts lateralized in the azimuthal plane (stationary or moving). In the present tests, the reference signals created stationary, intracranially centered percepts. At the onset of the respective deviant signal, its "location"

\section{REFERENCES}

Abel, S. M., Krever, E. M., and Alberti, P.W. (1990). Auditory detection, discrimination and speech processing in ageing, noise-sensitive and hearing-impaired listeners. Scand. Audiol. 19, 43-54.
Belin, P., McAdams, S., Thivard, L., Smith, B., Savel, S., Zilbovicius, M., Samson S., and Samson, Y. (2002). The neuroanatomical substrate of sound duration discrimination. Neuropsychologia 40, 1956-1964.

was also perceived as centered, but toward the end it appears to move (and vanish) to the left or right side (depending on the side of the signal with the shorter duration). According to the "snapshot theory" a motion is detected by the spatial difference of the targets onset and offset (Middlebrooks and Green, 1991; Grantham, 1995). Accordingly, the spatial position of onset and offset are interpolated and - given an appropriate disparity - a movement is perceived. In our tests both young subjects and most of the old subjects were able to perceive the "movement" and indicate its direction. However, older subjects performed worse than young adults. The cause might be a "binaural sluggishness" which becomes effective if the speed of interaural changes becomes too fast (Blauert, 1972). A reduction of the dynamics of interaural processing in old subjects (Grantham and Wightman, 1978) might be causal for their increased threshold values. Factors contributing to the increase in interaural duration discrimination values seem to be the reduction of the precision of interaural temporal integration of onset and offset coding of sounds at the IC and thalamic level due to failures in inhibition (Caspary et al., 2008; Walton, 2010).

So, it is conceivable that a deterioration of temporal processing in central auditory circuitries, will also result in a decrease in duration discrimination. These age-dependent changes point specifically to an abundant deterioration of central auditory processing in the elderly. We assume that both, the temporal processing in the nuclei of the auditory brainstem (Caspary et al., 2008; Walton, 2010) and at cortical processing stages (Snell and Frisina, 2000; Snell et al., 2002; Tremblay et al., 2007; Ross et al., 2010) might be affected by age and that these changes are behaviorally relevant (e.g., Gordon-Salant and Fitzgibbons, 1993; Schneider et al., 1994; Snell and Frisina, 2000; Snell et al., 2002; Tremblay et al., 2007).

\section{CONCLUSION}

The present study shows that older people exhibit increased JNDs in all tested acoustic features in comparison to the younger subjects. While the results were highly individual, the group data still disclosed patterns of impaired central auditory processing. Deteriorations in the auditory periphery are likely to affect processing in central auditory circuitries. While those deficiencies can be partly compensated by central processing, a decline in auditory temporal processing results in impairments which affect the whole central auditory system and its functionality. Elevations of JNDs in interaural frequency and duration discrimination point to an age-dependent decline of temporal processing mechanisms both at the level of the brainstem and in cortical auditory areas.

\section{ACKNOWLEDGMENTS}

Financial support for this work was supported by AHEAD [funded in part by EU project AHEAD (WP $4-\mathrm{AE}$ )] and International Max-Planck Research School "NeuroCom." Special thanks go to A. Ludwig, K. Schmiedchen, and I. Nitsche for their comments on an earlier version of the manuscript.

Biedermann, F., Bungert, P., Dorrscheidt, G. J., von Cramon, D. Y., and Rubsamen, R. (2008). Central auditory impairment in unilateral diencephalic and telencephalic lesions. Audiol. Neurootol. 13, 123-144.
Blauert, J. (1972). On the lag of lateralization caused by interaural time and intensity differences. Audiology 11, 265-270.

Blauert, J. (1997). Spatial Hearing: The Psychophysics of Human Sound 
Localization. Cambridge, MA: MIT Press.

Brant, L. J., and Fozard, J. L. (1990). Age changes in pure-tone hearing thresholds in a longitudinal study of normal human aging. J. Acoust. Soc. Am. 88, 813-820.

Bungert-Kahl, P., Biedermann, F., Dörrscheidt, G. J., von Cramon, D. Y., and Rübsamen, R. (2004). Psychoacoustic test tools for the detection of deficits in central auditory processing: normative data. $Z$. Audiol. 43, 48-71.

Buss, E., Hall, J. W., and Grose, J. H. (2004). Temporal fine-structure cues to speech and pure tone modulation in observers with sensorineural hearing loss. Ear Hear. 25, 242-250.

Cabeza, R., Anderson, N. D., Locantore, J. K., and McIntosh, A. R. (2002). Aging gracefully: compensatory brain activity in high-performing older adults. NeuroImage 17, 1394-1402.

Caspary, D. M., Ling, L., Turner, J. G., and Hughes, L. F. (2008). Inhibitory neurotransmission, plasticity and aging in the mammalian central auditory system. J. Exp. Biol. 211(Pt 11), 1781-1791.

Casseday, J. H., Fremouw, T., and Covey, E. (2002). “The inferior colliculus: hub of the auditory system," in Integrative Functions in the Mammalian Auditory Pathway, eds D. Oertel, R. R. Fay, and A. N. Popper (New York: Springer), 228-318.

CHABA. (1988). Speech understanding and aging. Working Group on Speech Understanding and Aging. Committee on Hearing, Bioacoustics, and Biomechanics, Commission on Behavioral and Social Sciences and Education, National Research Council. J. Acoust. Soc. Am. 83, 859-895.

Celik, O., Yalçin, S., Celebi,H., and Oztürk, A. (1996). Hearing loss in insulindependent diabetes mellitus. Auris Nasus Larynx 23, 127-132.

Craik, F. I. M., and Salthouse, T. A. (2000). The Handbook of Aging and Cognition. Mahwah, NJ: Erlbaum.

Cranford, J. L., Andres, M. A., Piatz, K. K., and Reissig, K. L. (1993). Influences of age and hearing loss on the precedence effect in sound localization. J. Speech Hear. Res. 36, 437-441.

Cruickshanks, K. J., Wiley, T. L., Tweed, T. S., Klein, B. E., Klein, R., MaresPerlman, J. A., and Nondahl, D. M. (1998). Prevalence of hearing loss in older adults in Beaver Dam, Wisconsin. The epidemiology of hearing loss study. Am. J. Epidemiol. 148, 879-886.

Divenyi, P. L., Stark, P. B., and Haupt, K. M. (2005). Decline of speech understanding and auditory thresholds in the elderly. J. Acoust. Soc. Am. 118, 1089-1100.
Dix, M. R., Hallpike, C. S., and Hood, J. D. (1948). Observations upon the loudness recruitment phenomenon, with especial reference to the differential diagnosis of disorders of the internal ear and VIII nerve. Proc. R. Soc. Med. 41, 516-526.

Dubno, J. R, Lee, F. S., Matthews, L. J., and Mills, J. H. (1997). Age-related and gender-related changes in monaural speech recognition. J. Speech Lang. Hear. Res. 40, 444-452.

Durlach, N. I., Thompson, C. L., and Colburn, H. S. (1981). Binaural interaction of impaired listeners. A review of past research. Audiology 20, 181-211.

Eckert, M. A., Walczak, A., Ahlstrom, J., Denslow, S., Horwitz, A., and Dubno, J. R. (2008). Age-related effects on word recognition: reliance on cognitive control systems with structural declines in speech-responsive cortex. J.Assoc. Res. Otolaryngol. 9, 252-259.

Felder, E., and Schrott-Fischer, A. (1995). Quantitative evaluation of myelinated nerve fibres and hair cells in cochleae of humans with age-related high-tone hearing loss. Hear. Res. 91, 19-32.

Fitzgibbons, P. J., and Gordon-Salant, S. (1995). Age effects on duration discrimination with simple and complex stimuli. J. Acoust. Soc. Am. 98, 3140-3145.

Fitzgibbons, P. J., and Gordon-Salant, S. (1998). Auditory temporal order perception in younger and older adults, 41, 1052-1060.

Folstein, M. F., Folstein, S. E., and McHugh, P. R. (1975). "Mini-mental state". A practical method for grading the cognitive state of patients for the clinician. J. Psychiatr. Res. 12, 189-198.

Fowler, E. P. (1936). A method for the early detection of otosclerosis: a study of sounds well above threshold. Arch. Otolaryngol. 24, 731-741.

Freyman, R. L., and Nelson, D. A. (1991). Frequency discrimination as a function of signal frequency and level in normal-hearing and hearingimpaired listeners. J. Speech Hear. Res. 34, 1371-1386.

Gates, G. A., Cooper, J. C., Kannel, W. B., and Miller, N. J. (1990). Hearing in the elderly: the Framingham cohort, 1983-1985. Part I. Basic audiometric test results. Ear Hear. 11, 247-256.

Gazzaley, A., Cooney, J. W., Rissman, J., and D'Esposito, M. (2005). Top-down suppression deficit underlies working memory impairment in normal aging. Nat. Neurosci. 8, 1298-1300.

Gibbon, J., Malapani, C., Dale, C. L., and Gallistel, C. (1997). Toward a neurobiology of temporal cognition: advances and challenges. Curr. Opin. Neurobiol. 7, 170-184.
Gordon-Salant, S. (2005). Hearing loss and aging: new research findings and clinical implications. J. Rehabil. Res. Dev. 42(4 Suppl. 2), 9-24.

Gordon-Salant, S., and Fitzgibbons, P. J. (1993). Temporal factors and speech recognition performance in young and elderly listeners. J. Speech Hear Res. 36, 1276-1285.

Gordon-Salant, S, Yeni-Komshian, G. Fitzgibbons, P. J., and Barrett, J. (2006). Age-related differences in identification and discriminationof temporal cues in speech segments. J. Acoust. Soc Am. 119, 2455-2466.

Grantham, D.W.(1995). "Spatial hearing and related phenomena," in Hearing, ed. B. C. J. Moore (London: Academic Press), 297-345.

Grantham, D. W., and Wightman, F. L. (1978). Detectability of varying interaural temporal differences. J. Acoust. Soc. Am. 63, 511-523.

Grothe, B., Pecka, M., and McAlpine, D. (2010). Mechanisms of sound localization in mammals. Physiol. Rev. 90, 983-1012.

Hasher, L., and May, C. (1999). "Inhibitory control, circadian arousal, and age," in Attention and Performance XVII: Cognitive Regulation of Performance: Interaction of Theory and Application, eds D. Gopher and A. Koriat (Cambridge: MIT Press), 653-675.

He, N., Dubno, R., and Mills, J. H. (1998). Frequency and intensity discrimination measured in a maximumlikelihood procedure from young and aged normal-hearing subjects. $J$. Acoust. Soc. Am. 103, 553-565.

He, N., Mills, H., and Dubno, J. R. (2007). Frequency modulation detection: effects of age, psychophysical method, and modulation waveform. J. Acoust. Soc. Am. 122, 467-477.

Heinrich, A., and Schneider, B. (2006) Age-related changes in within- and between-channel gap detection using sinusoidal stimuli. J. Acoust. Soc. Am 119, 2316-2326.

Humes, L. E. (1996). Speech understanding in the elderly. J. Am. Acad. Audiol. 7, 161-167.

Humes, L. E, Kewley-Port, D., Fogerty, D., and Kinney, D. (2010). Measures of hearing threshold and temporal processing across the adult lifespan. Hear. Res. 264, 30-40.

Ivry, R. B. (1996). The representation of temporal information in perception and motor control. Curr. Opin. Neurobiol. 6, 851-857.

Jennings, C. R., and Jones, N. S. (2001). Presbyacusis. J. Laryngol. Otol. 115, 171-178.

Johnson, M. L., Bengtson, V. L., Coleman, P. G., and Kirkwood, T. B. L. (2005). The Cambridge Handbook of Age and Ageing. Cambridge: Cambridge University Press.

Kaukoranta, E., Sams, M., Hari, R., Hämäläinen, M., and Näätänen, R (1989). Reactions of human auditory cortex to a change in tone duration. Hear. Res. 41, 15-21.

Koehnke, J., Culotta, C. P., Hawley, M. L. and Colburn, H. S. (1995). Effects of reference interaural time and intensity differences on binaural performance in listeners with normal and impaired hearing. Ear Hear. 16, 331-353.

König, E. (1957). Pitch discrimination and age. Acta Otolaryngol.48, 475-489.

Leek, M. R. (2001). Adaptive procedures in psychophysical research. Percept. Psychophys. 63, 1279-1292.

Ludwig, A. (2009). Psychoakustische und elektrophysiologische Untersuchungen zu zentral-auditiven Verarbeitungsstörungen während der Kindesentwicklung. Leipzig: Leipziger Universitätsverlag.

Marozeau, J., and Florentine, M. (2007). Loudness growth in individual listeners with hearing losses: a review. $J$. Acoust. Soc. Am. 122, EL81.

Mazelová, J., Popelar, J., and Syka, J. (2003). Auditory function in presbycusis: peripheral vs. central changes. Exp. Gerontol. 38, 87-94.

Mesulam, M. M. (1981). A cortical network for directed attention and unilateral neglect. Ann. Neurol. 10, 309-325.

Mesulam, M. M. (1998). From sensation to cognition. Brain J. Neurol. 121(Pt 6), 1013-1052.

Middlebrooks, J. C., and Green, D. M. (1991).Sound localization by human listeners. Annu. Rev. Psychol. 42, 135-159.

Mills, J. H., Dubno, J. R., and He, N. (1996). Masking by ipsilateral and contralateral maskers. J. Acoust. Soc. Am. 100, 3336-3344.

Nagel, I. E., Preuschhof, C., Li, S.-C., Nyberg, L., Bäckman, L. Lindenberger, U., and Heekeren, $\mathrm{H}$. R. (2009). Performance level modulates adult age differences in brain activation during spatial working memory. Proc. Natl. Acad. Sci. U.S.A. 106, 22552-22557.

Pichora-Fuller, M. K. (1997). Language comprehension in older listeners. J. Speech Lang. Pathol. Audiol. 21, 125-142.

Pichora-Fuller, M. K., and Souza, P. E. (2003). Effects of aging on auditory processing of speech. Int. J. Audiol. 42(Suppl. 2), 2S11-2S16.

Ross, B., Schneider, B., Snyder, J. S., and Alain, C. (2010). Biological markers of auditory gap detection in young, middle-aged, and older adults. PLoS ONE 5, e10101. doi: 10.1371/journal. pone.0010101

Salthouse, T. A. (1991). Age and experience effects on the interpretation 
of orthographic drawings of threedimensional objects. Psychol. Aging 6, 426-433.

Schneider, B. A., Pichora-Fuller, M. K., Kowalchuk, D., and Lamb, M. (1994). Gap detection and the precedence effect in young and old adults. $J$. Acoust. Soc. Am. 95, 980-991.

Schuknecht, H. F. (1955). Presbycusis. Laryngoscope 65, 402-419.

Snell, K. B., and Frisina, D. R. (2000). Relationships among age-related differences in gap detection and word recognition. J. Acoust. Soc. Am. 107, 1615-1626.

Snell, K. B., Mapes, F. M., Hickman, E. D., and Frisina, D. R. (2002). Word recognition in competing babble and the effects of age, temporal processing, and absolute sensitivity. J. Acoust. Soc. Am. 112, 720-727.

Soucek, S., Michaels, L., and Frohlich, A. (1986). Evidence for hair cell degeneration as the primary lesion in hearing loss of the elderly. J. Otolaryngol. 15, 175-183.

Tremblay, K., Picton, T. W., and Ross, B. (2007). Auditory evoked MEG responses to interaural phase changes: effects of aging on response latencies. Int. Congr. Ser. 1300, 69-72.

Turner, C. W., and Nelson, D. A. (1982). Frequency discrimination in regions of normal and impaired sensitivity. J. Speech Hear. Res. 25, 34-41.

Walton, J. P. (2010). Timing is everything: temporal processing deficits in the aged auditory brainstem. Hear. Res. 264, 63-69.

Woo, T.-U. W., Spencer, K., and McCarley, R. W. (2010). Gamma oscillation deficits and the onset and early progression of schizophrenia. Harv. Rev. Psychiatry 18, 173-189.

Zwislocki, J. J. (1972). A theory of central auditory masking and its partial validation. J. Acoust. Soc. Am. 52, 644-659.

Conflict of Interest Statement: The authors declare that the research was conducted in the absence of any commercial or financial relationships that could be construed as a potential conflict of interest.
Received: 15 December 2010; accepted: 19 April 2011; published online: 02 May 2011.

Citation: Freigang C, Schmidt L, Wagner J, Eckardt R, Steinhagen-Thiessen E, Ernst $A$ and Rübsamen R (2011) Evaluation of central auditory discrimination abilities in older adults. Front. Ag. Neurosci. 3:6. doi: 10.3389/fnagi.2011.00006

Copyright (c) 2011 Freigang, Schmidt, Wagner, Eckardt, Steinhagen-Thiessen, Ernst and Rübsamen. This is an openaccess article subject to a non-exclusive license between the authors and Frontiers Media SA, which permits use, distribution and reproduction in other forums, provided the original authors and source are credited and other Frontiers conditions are complied with. 